\title{
Numerical investigation of the effect of crosswind on sound propagation outdoors
}

\author{
Maarten Hornikx, \\ Eindhoven University of Technology, Building Physics and Services, P.O. Box 513, 5600 MB, Eindhoven, \\ The Netherlands \\ Timothy van Renterghem \\ Ghent University, Department of Information Technology, Sint-Pietersnieuwstraat 41, B 9000 Gent, \\ Belgium
}

\begin{abstract}
The effect of wind on sound propagation in the atmosphere has been studied extensively before with an emphasize on downwind sound propagation, typically representing worst-case scenarios. However, the influence of oblique and crosswind on propagation from various types of sources raises some questions in acoustic literature. In this work, the effect of a logarithmic wind speed profile at different wind directions has been studied for sound emitted by a point source, a coherent line source and an incoherent line source. For this purpose, a full three-dimensional wave-based method was used. For the incoherent line source simulation, the Harmonoise engineering approach based on a summation of source segments was considered as well and shows to be in satisfying agreement with the latter. While for a point source and coherent line source crosswind shows to have an insignificant effect, it is important in case of an incoherent line source. Also, the stretch of the incoherent line source contributing to the noise level at a receiver close to this line differs strongly depending on the wind direction.
\end{abstract}

\section{PACS}

43.20.El, 43.28.Fp, 43.28.Js 


\section{Introduction}

Sound propagation outdoors is strongly influenced by the refractive state of the atmosphere [1]. Phenomena linked to either downwind or upwind sound propagation have been studied extensively. Downwind sound propagation might lead to strongly increased sound levels relative to a still atmosphere at distant receivers. Upwind from a source, very low sound levels are to be expected due to the formation of an acoustic shadow zone. However, the effect of wind directions other than purely upwind or downwind (which will here be referred to generally as side wind or oblique wind, and crosswind to indicate a wind direction parallel to a line source or orthogonal to the plane containing point source and receiver) on sound propagation outdoors is less clear.

Wind (at any direction) is able to significantly influence the vertical temperature stratification in the atmosphere. Typically, the build-up of ground-based temperature inversion conditions will be strongly counteracted due to the enhanced mixing compared to a still atmosphere. In addition, shear-induced wind turbulence will lead to coherence loss, typically resulting in less pronounced destructive interference dips in outdoor sound propagation [2]. As these wind effects are expected for any wind direction, side wind will influence the propagation of sound in the atmosphere at least indirectly.

Regarding the direct effect of oblique wind and crosswind, most authors mention that in case of a point source, wind components normal to the propagation direction could be neglected. Rasmussen [3] expected that such a wind component only has a limited influence on sound propagation from a point source. Yoshihisa [4] mentioned that in his experiment with a loudspeaker considered as a point source, vector wind (i.e. the wind speed vector projected on the plane through source and receiver) is a sufficient parameter for an accurate prediction of the refraction effect. The CONCAWE [5] meteorological classification model, developed for use in relation to industrial noise sources (and thus point sources for distant receivers), treats strong oblique winds in a similar way as moderate downwind propagation, while no wind and crosswind are put in the same category [6]. Li and Wang [6] concluded, based on their analytical considerations, that the effect of wind can be ignored in the crosswind situation because the effective Mach number is zero under such circumstances. Cheng et al. [8] reported that in crosswind directions, there is no acoustic refraction in the vertical direction, and the sound pressure level is thus "only slightly" affected by the wind. By means of 2D and 3D Fast Field Program (FFP) calculations, Salomons [1] found that crosswind has no effect on sound propagation from a point source and concluded that 2D modelling, so neglecting crosswind, is accurate. An exception has to be made when there is interaction between the terrain and the wind flow, e.g. in case of a point source located on top of an isolated hill as discussed by Blumrich and Heimann [9]. In that case, neglecting side wind (by applying the effective sound speed approach as tested in their work) could lead to errors of several dBs. 
In case of line sources, the reported effects of oblique and crosswind diverge. In case of the aforementioned experiment by Yoshihisa [4], considering a real traffic (line) source, it was mentioned that both vector wind speed and wind direction were needed for good predictions. Oshima and li [10] concluded that the effects of the parallel component of the wind velocity on sound propagation were unclear in their experiment and required further investigation. It was also mentioned that the refractive effects of crosswind are usually neglected, but that this could be of relevance for infinite line sources, either being coherent or incoherent [10]. Tanaka and Shiraishi [11] concluded that vector wind was sufficient to predict meteorological effects of sound propagation from roads, but considered a case with mainly downwind sound propagation and aimed at long-term averaged levels, where the downwind episodes dominate. Makarewicz [12] concluded that for the special case of wind blowing along a train track, only a (small) segment of the track contributes to the noise at a receiver. This can be explained because parts of the track are located either upwind or downwind relative to the receiver. Implicitly, the fact that tree rows behind a highway noise barrier limit screen-induced refraction of sound by wind not only for a wind direction normal to the road, but also for oblique wind directions, indicates that such oblique winds affect sound pressure levels for a fixed receiver [13]. The Harmonoise reference model [14] proposed to break up traffic line sources in small segments, and uses point source calculations from the middle of each segment towards the receiver. In a final step, all contributions should be added incoherently. The wind vector projected on the plane between each road point source and receiver should be considered. As a result, source points further away from the receiver take an increasingly growing part of the wind vector into account. It can therefore be concluded that crosswind cannot be simply neglected in case of (incoherent) line sources.

The purpose of current work is to demonstrate the effect of both oblique wind and crosswind for scenarios consisting of a point source, a coherent line source and an incoherent line source; threedimensional full-wave numerical calculations were used. The validity of 2D approaches to model sound propagation from a point source and incoherent line source in oblique and crosswind conditions are numerically verified. For an incoherent line source in wind, additional analysis is made to reveal the contributing part arriving at a receiver at close distance for various wind directions.

\section{Scenarios and methodology}

Figure 1 displays the studied 3D scenarios. All scenarios concern atmospheric sound propagation over a rigid or soft ground surface.. Three different source types have been investigated: a coherent line source, an incoherent line source and a point source. The coherent line source case was chosen because it mimics the more often studied 2D cases. The incoherent line source is representative for noise from a

busy road, and the point source solution is relevant for industrial noise sources and as the fundamental result from which the incoherent line source solution can be composed.

The receiver is located at $50 \mathrm{~m}$ from the source(s). Two receiver heights have been chosen, $1.5 \mathrm{~m}$ and $4.0 \mathrm{~m}$. The atmospheric wind components only depend on the vertical coordinate and are computed as: 
$u_{x}(z)=b \ln \left(1+\frac{z}{z_{0}}\right) \cos (\alpha)$,

$u_{y}(z)=b \ln \left(1+\frac{z}{z_{0}}\right) \sin (\alpha)$,

with $\boldsymbol{u}=\left[u_{x}, u_{y}\right]$ the atmospheric wind velocity vector with components in $x$ and $y$ direction respectively, where $x$ is the direction perpendicular to the line source, $y$ the direction parallel to the line source and $z$ the vertical direction; $b=2 \mathrm{~m} / \mathrm{s}$ corresponding to a very strong wind, $z_{0}=0.1 \mathrm{~m}$ the roughness length and $\alpha$ the angle of the wind direction relative to the direction from source to receiver. This logarithmical wind speed profile is representative for a neutral atmosphere. The large value for the friction velocity $b$ was chosen to ensure pronounced wind effects already at short distances to illustrate the effect of source type and its approximation, the latter being the main goal of this work.

Three-dimensional sound propagation in a moving atmosphere can be computed by solving the linearized Euler equations. Several numerical techniques qualify for solving these equations. In current work, it was opted to use the Fourier pseudospectral time-domain (PSTD) methodology following [15]. This method combines a high accuracy with a relatively low computational cost, as only two spatial points are needed per acoustic wavelength and is well suited to model sound propagation over rigid soil in an unbounded domain. Details of the PSTD method can be found in literature [15]. For current work, a spatial mesh with an equidistant spacing of $d x=0.065 \mathrm{~m}$ was used. This allows computing sound propagation up to the $2500 \mathrm{~Hz} 1 / 3$ octave band. Acoustic energy is entering the domain by the following pressure source function:

$s(t)=\sin \left(2 \pi f_{s} / 8\left(t_{0, y}-t\right)\right) e^{-3 \cdot 10^{-6}\left(\frac{f_{s}}{4000}\right)^{2}\left(t_{0, y}-t\right)^{2}}$,

with $f_{s}=c_{0} / d x$ the spatial sampling frequency, $c_{0}$ the adiabatic speed of sound for which a value of 340 $\mathrm{m} / \mathrm{s}$ has been used in the calculations, and $t_{0, y}$ the center time of the source function. In PSTD, acoustic velocity components are spatially staggered with the acoustic pressure components, and the acoustic velocity components located at the ground surface are set to zero. The sources are located at $z_{s}=d x / 2=$ $0.0325 \mathrm{~m}$ above the ground surface, which could represent noise emitted from the interaction between vehicle tyres and the road surface. In PSTD, the domain was modelled by a slice of the physical domain, see Figure 2 . The boundaries in the $z$ - and $x$-direction were truncated by a perfectly matched layer (PML) to obtain a reflection free boundary. In $y$-direction, the boundaries were modelled as periodic.

For the point source, $y_{s}=65 / 2 d x$ was used. For the coherent line source, the source function (2) was applied to all grid positions in $y$-direction, i.e. $y_{s}=1 / 2 d x, 3 / 2 d x, 5 / 2 d x, \ldots, 127 / 2 d x$, with $t_{0, y}=0.01 \mathrm{~s}$. For the incoherent line source, the source function (2) was also applied to all grid positions in $y$-direction, but with $t_{0, y}$ a random value for all grid positions between 0.01 and $0.06 \mathrm{~s}$. By imposed different center times, an incoherent source is obtained. This approach was used earlier [16]. Calculations for PS (point source) and CLS (coherent line source) have ran with a length of 2000 discrete samples (about $0.19 \mathrm{~s}$ ), which ensured convergence of the computed sound pressure levels. For the ILS (incoherent line source), the length of the calculation is related to the length of the actual line source. A total signal length of 
8000 samples was used $(0.76 \mathrm{~s})$, corresponding to a total line source length of about $500 \mathrm{~m}$. For the PS and CLS, results at the receiver position at $y_{r}=65 / 2 d x$ were used. For the ILS results, the acoustic energy has first been averaged over all receiver positions from $1 / 2 d x$ to $127 / 2 d x$ before computing the level difference. The reason for doing so is that the adopted incoherent line source approach still leads to some coherent effects when only one receiver position is taken as the line source has a periodicity of $64 d x$. This is reduced when averaging over more positions.

An additional calculation was performed for an incoherent line source following the procedure described in the Harmonoise reference sound propagation model [14]. The line source is split up in a number of segments, where sound propagation is calculated in 2D vertical planes through the centers of these segments and the receiver. Such a set of $2 \mathrm{D}$ calculation implies sound propagation from a coherent line source, orientated normal to the aforementioned propagation plane. The contributions from the different line segments were then summed together incoherently. This approach relies on the fact that sound propagation, expressed relative to free field sound propagation, is very similar for both a point source and a coherent line source [17]. This approach avoids the need for a full 3D sound propagation model. The wind vector is projected on the propagation plane; consequently, the vector wind speed changes from segment to segment. The sound propagation calculations were performed with the Green's Function Parabolic Equation method (GFPE) [1], accounting for refraction in detail, still allowing reasonably large propagation steps in the propagation direction. In vertical direction, one tenth of the wavelength is needed as spatial discretization step. GFPE uses the effective sound speed approach, which is accurate in the current case. For segments involving upwind sound propagation, no corrections for turbulent scattering were implemented, as these will be largely dominated by segments involving downwind propagation towards the receiver.

Convergence of the summed contributions from all segments at each $1 / 3$ octave band was found using the following values: $800 \mathrm{~m}$ total length of the line source, $10 \mathrm{~m}$ segment length and 5 times the wavelength for the propagation step in forward direction in the PE model. A sufficiently thick damping layer was applied at the top of the simulation domain, where the damping parameters were made frequency dependent following the practical findings as found in [1]. Twenty frequencies were calculated for each $1 / 3$-octave band.

\section{Results}

Figure 3 shows $\Delta L$, i.e. the sound pressure level in an atmosphere with a wind field relative to a still atmosphere as computed by

$\Delta L=10 \log _{10}\left(\frac{\sum_{y_{r}}\left|P_{\text {wind }}\left(\boldsymbol{x}_{r}\right)\right|^{2}}{\sum_{y_{r}}\left|P_{\text {still }}\left(\boldsymbol{x}_{\boldsymbol{r}}\right)\right|^{2}}\right)$,

with

$P_{\text {wind }}\left(\boldsymbol{x}_{\boldsymbol{r}}\right)=\mathcal{F}_{t}\left(p_{\text {wind }}\left(\boldsymbol{x}_{\boldsymbol{r}}, t\right)\right)$, 
$P_{\text {still }}\left(\boldsymbol{x}_{\boldsymbol{r}}\right)=\mathcal{F}_{t}\left(p_{\text {still }}\left(\boldsymbol{x}_{\boldsymbol{r}}, t\right)\right)$,

where $y_{r}=65 / 2 d x$ for the PS and CLS cases and $y_{r}=1 / 2 d x, 3 / 2 d x, 5 / 2 d x, \ldots, 127 / 2 d x$ for the ILS cases, $\mathcal{F}_{t}$ is the forward Fourier transform and $p_{\text {wind }}$ and $p_{\text {still }}$ are the recorded pressure time signals for the situations with or without wind respectively. Positive values indicate that larger levels are predicted when wind is present. In case of the GFPE calculation implementing the Harmonoise approach, the averaging over many receiver positions was not performed. Results from the full 3D PSTD calculations for three wind angles are plotted in one subfigure and plots have been made for the three source types and two receiver heights. Furthermore, for displaying the effect of wind at larger distances, Figure 4a) and $b$ ) contain results for the CLS for a receiver located at a distance of $250 \mathrm{~m}$ from the source. The results in these subfigures were computed with the 2D PSTD method, which is computationally less demanding than the 3D method. Figure 4c) and d) include ILS results from using the Harmonoise approach.

Most results show values lower than $0 \mathrm{~dB}$ for the higher frequencies. In downwind conditions, levels higher than $0 \mathrm{~dB}$ can be expected. However, it is known from literature (e.g. Figure 4.24 from [1]) that this expected effect is more pronounced for larger source to receiver distances. This is also confirmed by the upper lines in Figures 4a) and b) for a receiver located at a distance of $250 \mathrm{~m}$ from the source.

The results from the point source (PS) and coherent line source (CLS) are similar, and are less smooth over frequency for the lower receiver position: several 'sound waves' have arrived leading to distinct interference effects (see e.g. Figure 4.7 from [1]). At the higher position at the other hand, only a direct sound wave and the low frequency part of a ground reflected sound wave have arrived (this was visible in the time signals of the PSTD calculations). The direct sound wave has a lower amplitude than the sound wave in the absence of wind, due to the diverging effect of the refracting waves (see section L.3.6 from [1]). This results in negative $\Delta L$ values for the high frequencies. The contribution from the ground reflected wave leads to higher values for the low frequencies and a weak interference effect around 400 $\mathrm{Hz}$.

Both PS and CLS results show no wind effect for $\alpha=90$ deg. The PS and CLS results are similar to the 2D PSTD results from Figures $4 a$ ) and $b$ ).

The results from the incoherent line source (ILS) deviate from the PS and CLS results. For the lower receiver position, the interference effects for wind angles $\alpha=0$ deg and $\alpha=60$ deg are much less pronounced, caused by the incoherent effect of the multiple sound sources forming the line source. Also, in contrast to the PS and CLS results, the wind effect for $\alpha=90$ deg cannot be neglected for the ILS results. In contrast, results for $\alpha=90 \mathrm{deg}$ are almost similar to the $\alpha=60 \mathrm{deg}$ results. This can be seen for both receiver positions. These results confirm that the effect of crosswind in the case of an incoherent line source cannot be neglected for the studied configuration, even if the direction of the wind is perpendicular to the plane connecting the receiver and line source.

Results from the Harmonoise ILS approach agree quite well with the PSTD results as shown in Figures 4c) and d). The average difference amounts to $0.6 \mathrm{~dB}$, computed as the arithmetic average of the absolute differences in $1 / 3$ octave band values for all scenarios as plotted in Figures $4 c$ ) and d). These slight 
differences can be attributed to the approximate way of computing the ILS with PSTD as reported in [17] and the approach of adding finite-length segments in the Harmonoise method. Regarding the first aspect, the $y$-periodicity in the PSTD prohibits full incoherence, as well as the limited center time range of the source functions. Concerning the latter aspect, the road has been discretized in $10 \mathrm{~m}$ wide segments.

The Harmonoise approach was also used to compute the level difference of the ILS in the presence of a soft ground surface (grassland approach by an effective flow resistivity of $150 \mathrm{kPa} \mathrm{s} / \mathrm{m}^{2}$ and the surface impedance computed with the Delany and Bazley model [18]). Results are plotted in Figure 5. For the lowest receiver position, the interference dip due to multiple waves is shifted down in the frequency range due to additional phase delays upon interaction with the ground surface. The dip is more pronounced in the soft ground case compared to the rigid ground case. At high frequencies, the level difference is larger for the soft ground case. This can be explained by the lower amount of ground interaction of the sound waves that have reflected with the ground surface at least one time, compared to the grazing ground interaction of the direct sound wave that leads to high damping, being the only contributing sound wave in the case without wind. For the $4 \mathrm{~m}$ high receiver position, similar differences between the rigid and soft ground cases take place. As the contribution from ground reflected waves is lower for the $4 \mathrm{~m}$ receiver position, the effects are more moderate. Differences between the wind directions modelled can be observed, but the same trends are obtained. As regards the level difference due to crosswind, $1 / 3$ octave band differences of 3.4 and $7.8 \mathrm{~dB}$ are observed for the lower receiver position for respectively the hard and soft ground cases.

The ILS calculations using the Harmonoise approach, calculated with GFPE, allow further analysis of the relevance of specific segments in function of frequency and wind direction, which is shown in Figures 6 and 7 for the configuration with a rigid ground and a receiver height of $1.5 \mathrm{~m}$. The plotted quantities are the levels relative to the maximum level of the configuration without wind, expressed as SPL in $d B$. In case of no-wind or a wind direction normal to the line source ( $0 \mathrm{deg})$, contributions from segments are fully symmetrical around the receiver position. In case of oblique wind (60 deg) or crosswind (90 deg), the lower part of the line source, i.e. $y_{s}<0 \mathrm{~m}$, contributes significantly more than the upper part, the latter being characterized to a large degree by upwind sound propagation conditions towards the receiver.

The plots in Figures 6 and 7 show a complex behavior. In absence of wind, segments closest to the receiver lead to the strongest contributions, independent of frequency for this particular case of a rigid ground plane. Contributions from other segments become rapidly minor when moving away from the receiver along the source line due to spherical divergence. In case of 0 deg wind direction and up to 500 $\mathrm{Hz}$, the SPL difference with the case without wind increases with frequency for all line source segments, see Figure 7, and the difference is larger for the further line source segments. The latter can be attributed to the number of refraction-induced ground reflected waves that increases with distance. For frequencies above $500 \mathrm{~Hz}$, SPL values of the line source segments for short $y_{s}$-positions drop below the case without wind due to the negative interferences between multiple sound waves. These 
interferences also occur for line source segments for further $y_{s}$-positions, but these SPL levels do not drop below the results without wind due to the higher number of sound waves.

In case of oblique or crosswind, the SPL values up to $500 \mathrm{~Hz}$ are even larger than for the case of 0 deg wind direction (for $y_{s}<0 \mathrm{~m}$ ), as even more multiple ground reflected sound waves reach the receiver position due to a higher wind speed component for line source segments located at further $y_{s}$-positions. For $y_{s}>0 \mathrm{~m}$, only very low frequencies still contribute to the receiver due to upwind refraction. High frequency contributions coming from segments with $y_{s}>0 \mathrm{~m}$ are nearly absent. The results of Figure 7 clearly indicate that for the octave bands up to $500 \mathrm{~Hz}$, the total level of the incoherent line source for the oblique and crosswind cases exceed the no wind case level, a result which was already shown in Figure 3.

Finally, an analysis of the relevant length of the line source is summarized in Figure 8, for both the receiver at $1.5 \mathrm{~m}$ and $4 \mathrm{~m}$ height and for the rigid and soft ground surfaces. As a criterion, the total level from the excluded one-sided line source segments is $10 \mathrm{~dB}$ lower than the total line source level. The slowest converging full octave band was chosen for the shown stretch of the road. The segment length is taken to be $10 \mathrm{~m}$ and stays the same over the full length of the incoherent line source. Note that the shown stretches hold for these specific criteria.

In case of no wind, a receiver height if $1.5 \mathrm{~m}$ and a rigid ground surface, the relevant part of the line source is situated between $y_{s}=-120 \mathrm{~m}$ and $y_{s}=120 \mathrm{~m}$. In case of wind normal to the line source, this strongly shifts to $y_{s}=-270 \mathrm{~m}$ and $y_{s}=270 \mathrm{~m}$. In case of oblique or crosswind, symmetry in contributions is not present anymore. The contributions from the line segments that are governed by upwind sound propagation are limited $\left(y_{s}<=50 \mathrm{~m}\right)$, and somewhat larger parts of the line source are relevant than for a perpendicular wind at negative $y_{s}$. For the same receiver height but for the soft ground surface, the same stretch is found for the case without wind. However, the stretches are smaller in the three cases with wind.

For the $4 \mathrm{~m}$ receiver height, the stretches for the wind cases are somewhat smaller than for the $1.5 \mathrm{~m}$ receiver height, both for the rigid as for the soft ground surface. This is in line with Figures 3-5, showing that the influence of wind is larger for the lower receiver height.

\section{Conclusions}

In this work, numerical calculations were carried out to study the effect of an atmospheric wind speed profile on propagation of sound from various source types in the presence of a rigid or soft ground surface: a point source, a coherent line source and an incoherent line source. In particular, the direction of the wind field with respect to source and receiver is of interest. A receiver position at $50 \mathrm{~m}$ from the (line) source was considered. As regards the point source and the coherent line source, the wind effect is relevant for normal and oblique wind directions, and similar for both source types. On the contrary, for crosswind, i.e. the wind direction parallel to a coherent line source or orthogonal to the plane 
containing point source and receiver, no wind effect is predicted. Furthermore, it was found that a 2D calculation approach with the projected wind velocity is similar to the $3 \mathrm{D}$ calculations with a point source. As regards the incoherent line source, although the contribution of line source segments is quite different for normal, oblique and crosswind directions, the total wind effect of these scenarios follows a similar trend. It can be concluded that the crosswind effect cannot be neglected for the studied configurations, with wind effects up to $7.8 \mathrm{~dB}$ ( $1 / 3$ octave band value). Note that the incoherent line source results are most important, as this case resembles noise propagation from busy road traffic. Results show that the wind effect for an incoherent line source as computed with the Harmonoise reference approach is very similar to results from using a full 3D wave-based technique, with an average difference of $0.6 \mathrm{~dB}$, taking into account these small differences by using different numerical techniques.

Finally, it was found that part of the incoherent line source that influences the sound pressure level at the receiver location largely changes with wind direction, especially at the lower receiver height and rigid ground surface. In case of a dominant wind direction relative to the road's length axis at a specific location, focus on specific parts of the road when implementing noise action plans (e.g. applying silent road surfaces) makes sense from an economical point of view.

\section{References}

[1] E. Salomons: Computational atmospheric acoustics. Dordrecht, Kluwer, 2001.

[2] E. Salomons, V. Ostashev, S. Clifford, R. Lataities: Sound propagation in a turbulent atmosphere near the ground: An approach based on the spectral representation of refractive-index fluctuations. Journal of the Acoustical Society of America 109 (2001) 1881-1893.

[3] K. Rasmussen: Outdoor sound propagation under the influence of wind and temperature gradients. Journal of Sound and Vibration 104 (1986) 321-335.

[4] K. Yoshihisa: Difference between the effect of wind on sound-propagation from a point-source and from a line source. Noise Control Engineering Journal 23 (1984) 104-104.

[5] K. Marsh: The CONCAWE model for calculating the propagation of noise from open-air industrialplants. Applied Acoustics 15 (1982) 411-428.

[6] K. Attenborough, K. Li, K. Horoshenkov: Predicting outdoor sound. London and New York, Taylor and Francis, 2007.

[7] K. Li, Q. Wang: Analytical solutions for outdoor sound propagation in the presence of wind. Journal of the Acoustical Society of America 102 (1997) 2040-2049.

[8] R. Cheng, P. Morris, K. Brentner: A three dimensional parabolic equation method for sound propagation in moving inhomogeneous media. Journal of the Acoustical Society of America 126 (2009) 1700-1710.

[9] R. Blumrich, D. Heimann: Numerical estimation of atmospheric approximation effects in outdoor sound propagation modelling. Acta Acustica united with Acustica 90 (2004) 24-37.

[10]T. Oshima T, M. li: Field measurements on wind effects to propagation of road traffic noise over open and flat ground. Applied Acoustics 74 (2013) 141-149. 
[11]S. Tanaka, B. Shiraishi: Wind effects on noise propagation for complicated geographical and road configurations. Applied Acoustics 69 (2008) 1038-1043.

[12]R. Makarewicz: Impact of refraction on train noise. Applied Acoustics 57 (1999) 279-287.

[13]T. Van Renterghem, D. Botteldooren: Effect of a row of trees behind noise barriers in wind. Acta Acustica united with Acustica 88 (2002) 869-878.

[14]J. Defrance, E. Salomons, I. Noordhoek, D. Heimann, B. Plovsing, G. Watts, H. Jonasson, X. Zhang, E. Premat, I. Schmich, F. Aballea, M. Baulac, F. de Roo: Outdoor sound propagation reference model developed in the European Harmonoise project. Acta Acustica united with Acustica 93 (2007) 213227.

[15]M. Hornikx, R. Waxler, J. Forssén: The extended Fourier pseudospectral time-domain method for atmospheric sound propagation. Journal of the Acoustical Society of America 128 (2010) 1632-1646.

[16]M. Hornikx, J. Forssén: Modelling of sound propagation to three-dimensional urban courtyards using the extended Fourier PSTD method. Applied Acoustics 72 (2011) 665-676.

[17]T. Van Renterghem, E. Salomons, D. Botteldooren: Efficient FDTD-PE model for sound propagation in situations with complex obstacles and wind profiles. Acta Acustica united with Acustica 91 (2005) 671-679.

[18]M. Delany, E. Bazley: Acoustical properties of fibrous absorbent materials. Applied Acoustics 3 (1970) 105-116. 


\section{Top view}
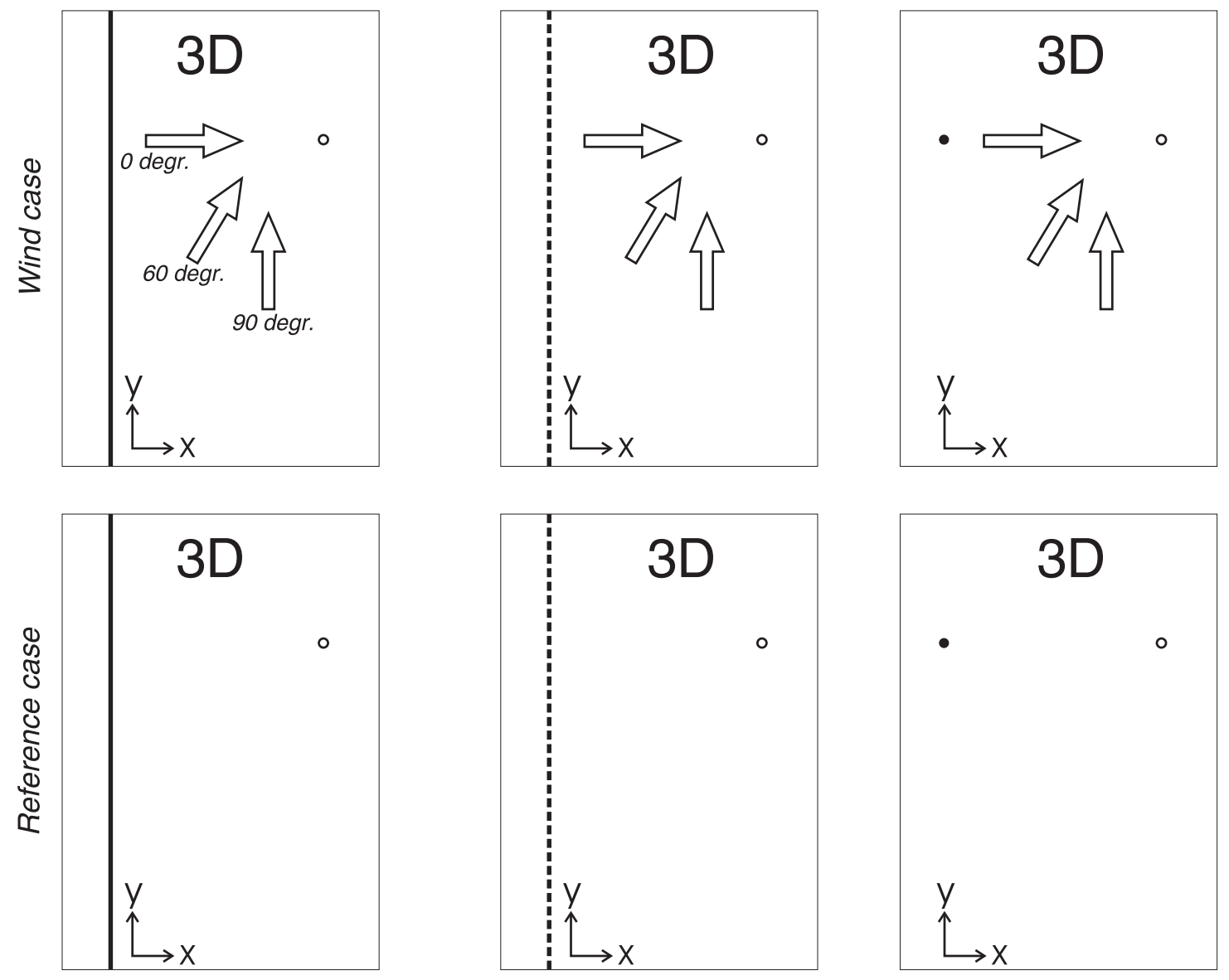

\section{Cross section}
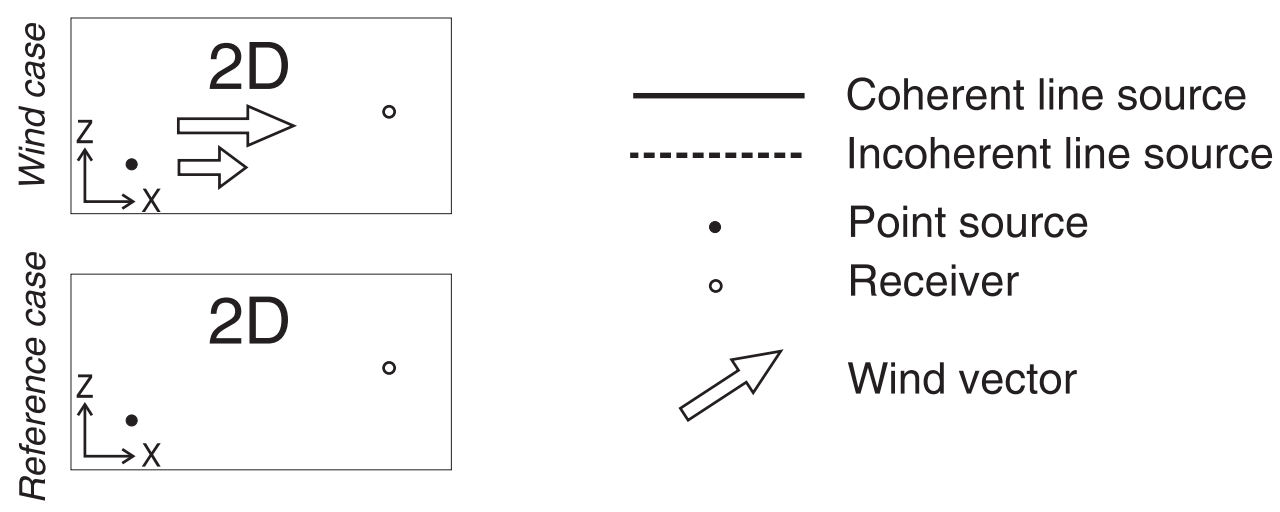

Figure 1. Scenarios studied: sound propagation from three different source types, for three different wind scenarios. Simulations are either carried out with a full 3D model (see Figure 2), or from 2D cross sections with projected wind components. The ground surface is either rigid or soft (Delany and Bazley model [18] with a flow resistivity of $150 \mathrm{kPa} \mathrm{s} / \mathrm{m}^{2}$ ). 


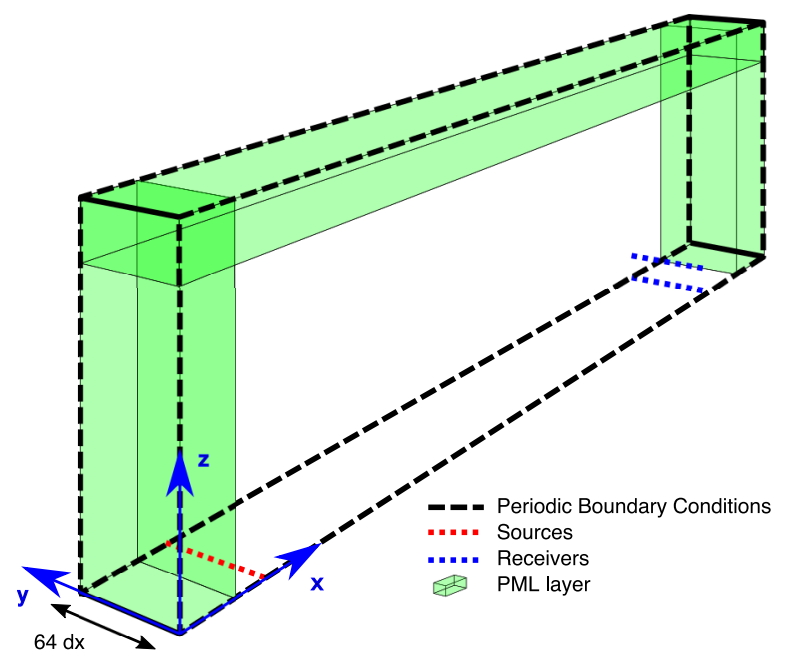

Figure 2. Numerical domain used to compute sound propagation with the PSTD method.
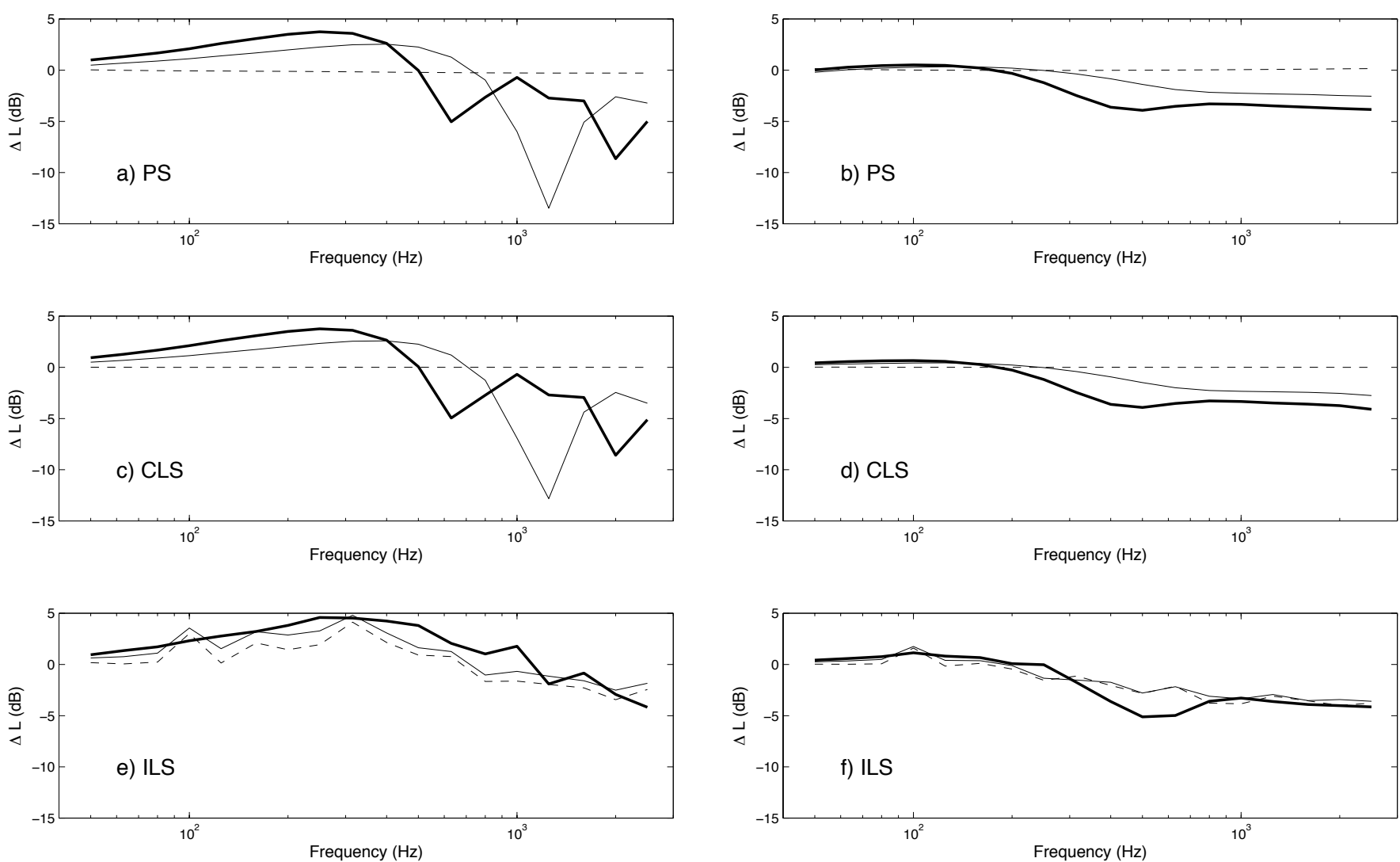

Figure 3. Relative sound pressure levels for receiver distance $x_{r}=50 \mathrm{~m}$ and receiver heights $z_{r}=1.5 \mathrm{~m}$ (left plots) and $z_{r}=4 \mathrm{~m}$ (right plots) from full 3D PSTD calculations. Results are shown for a point source (PS), coherent line source (CLS) and incoherent line source (ILS). (solid thick) wind angle $\alpha=0 \mathrm{deg}$, (solid thin) wind angle $\alpha=60 \mathrm{deg}$ and (dashed) wind angle $\alpha=90 \mathrm{deg}$. 

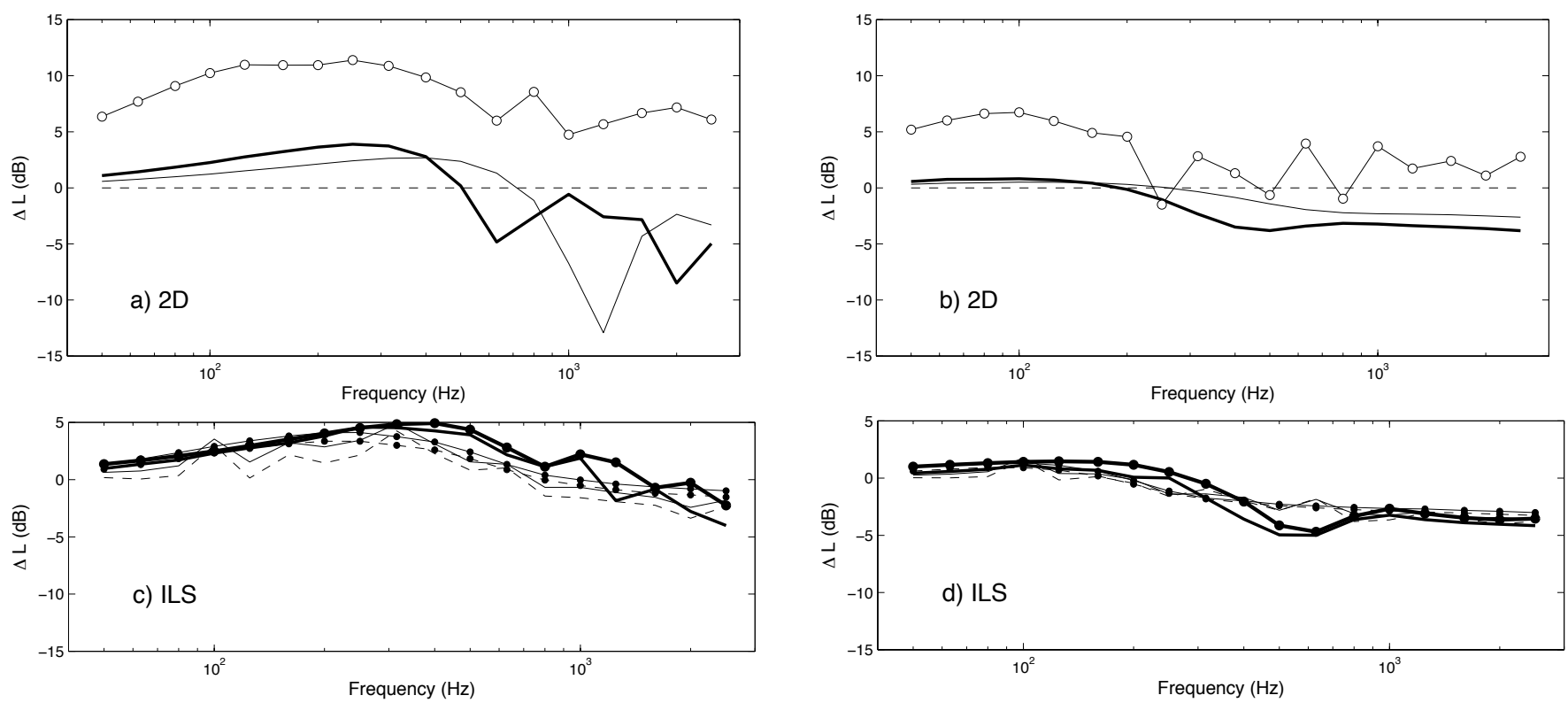

Figure 4. Relative sound pressure levels for receiver heights $z_{r}=1.5 \mathrm{~m}$ (left plots) and $z_{r}=4 \mathrm{~m}$ (right plots). a) and b) 2D PSTD calculations. (solid thick) wind angle $\alpha=0 \mathrm{deg}$, (solid thin) projected wind component from angle $\alpha=60$ deg, (dashed) projected wind component from angle $\alpha=90 \mathrm{deg}$ and (solid thin with circles) wind angle $\alpha=0$ deg with receiver at $250 \mathrm{~m}$ distance; c) and d) (lines) 3D PSTD calculations and (lines with circles) the HN approach.
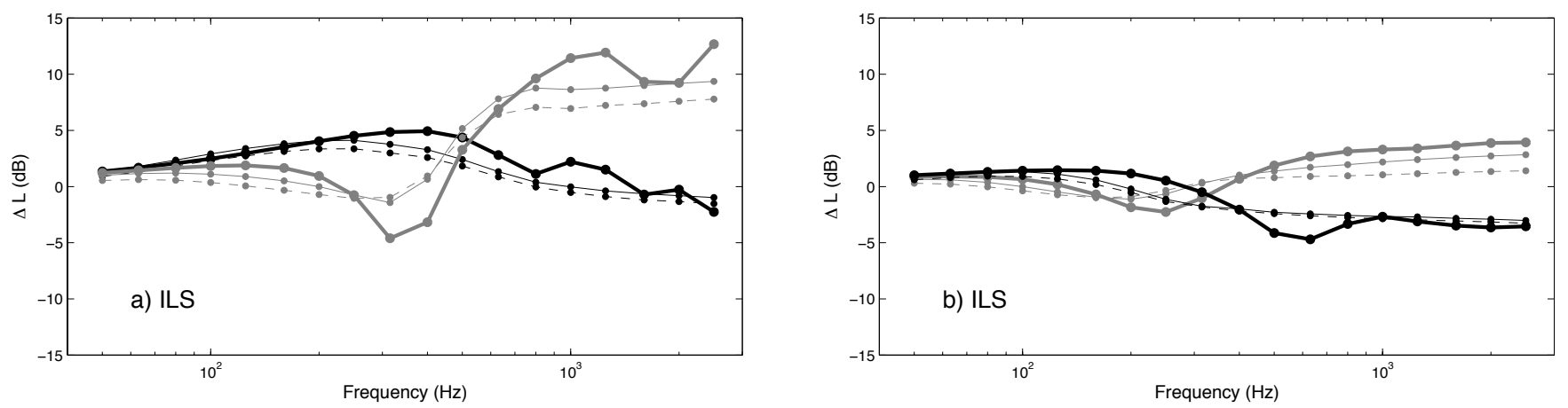

Figure 5. Relative sound pressure levels for receiver heights a) $z_{r}=1.5 \mathrm{~m}$ and b) $z_{r}=4 \mathrm{~m}$ computed with the HN approach. Black lines with circles: rigid ground surface (repeated from Fig. 4c and 4d), Grey lines with circles: soft ground surface. 
a)
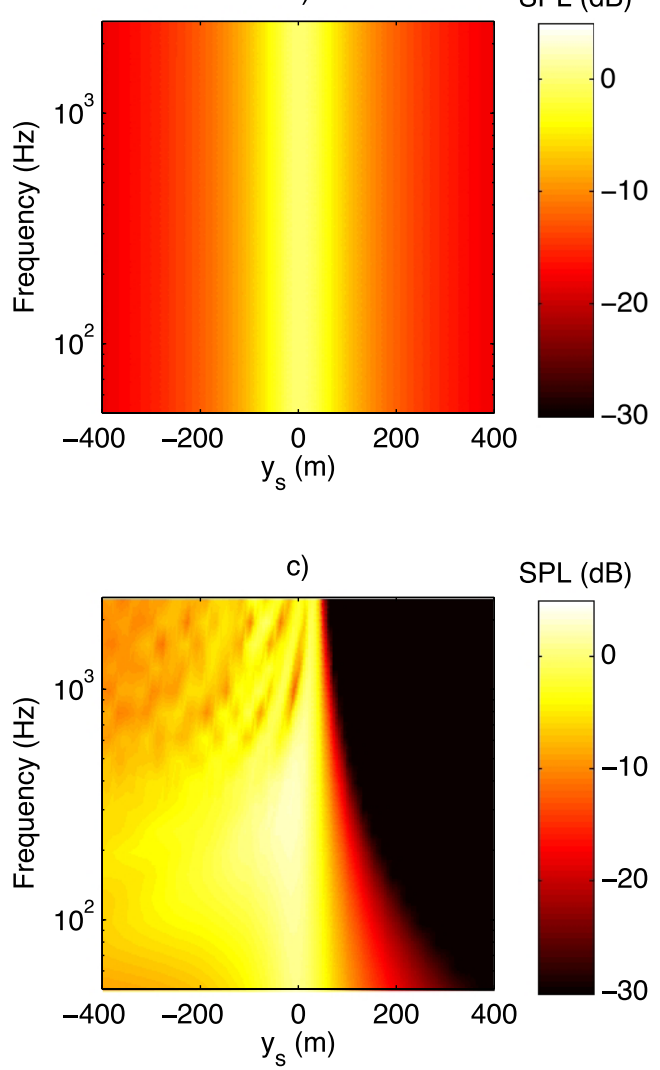

b)

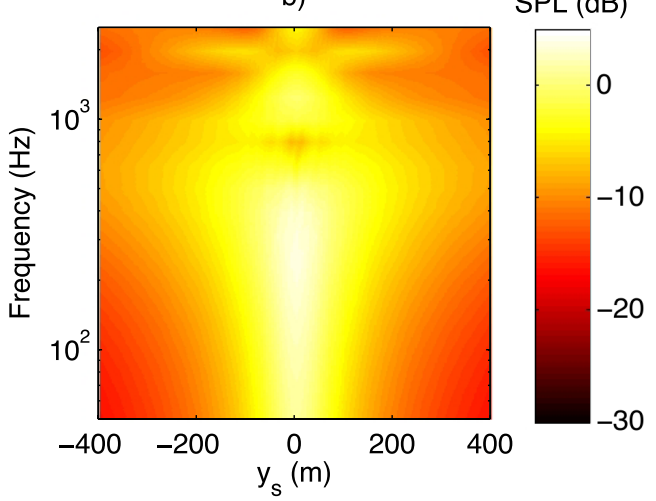

d)

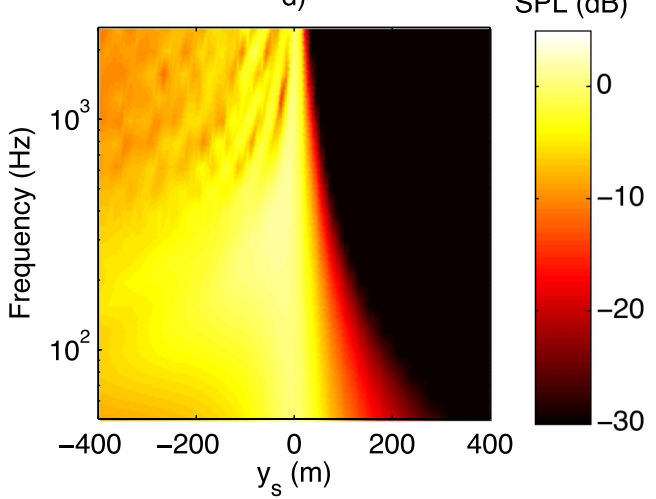

Figure 6. Contributions from the different ILS line segments with centers at $y_{s}$, relative to the maximum contribution at all $1 / 3$ octave bands from $50 \mathrm{~Hz}$ to $2.5 \mathrm{kHz}$ for the configuration without wind, for a) absence of wind, b) a wind direction normal to the incoherent line source (0 deg), c) oblique wind direction (60 deg) and d) crosswind (90 deg). The receiver is at $\left(x_{r}, y_{r}\right)(50,0)$. The receiver height is $1.5 \mathrm{~m}$ and the ground surface is rigid. 

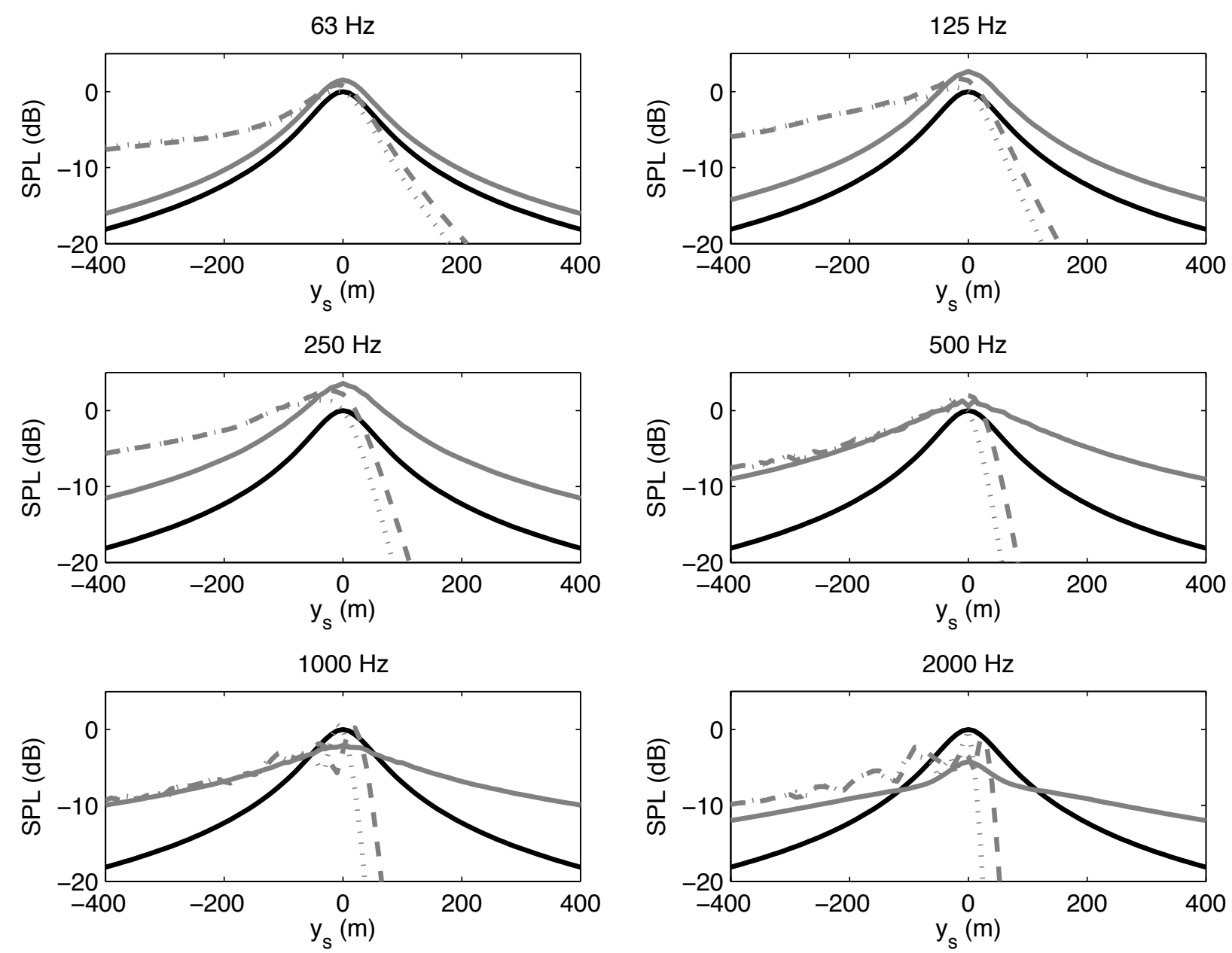

Figure 7. Contributions from the different ILS line segments with centers at $y_{s}$, split up in separate graphs for each octave band considered. Levels are referred to the maximum level for the case without wind. The receiver is at $\left(x_{r}, y_{r}\right)(50,0)$. The receiver height is $1.5 \mathrm{~m}$ and the ground surface is rigid. Black: no wind case, Solid grey: 0 deg wind angle, Dashed grey: 60 deg wind angle, Dotted grey: 90 deg wind angle. 
a)

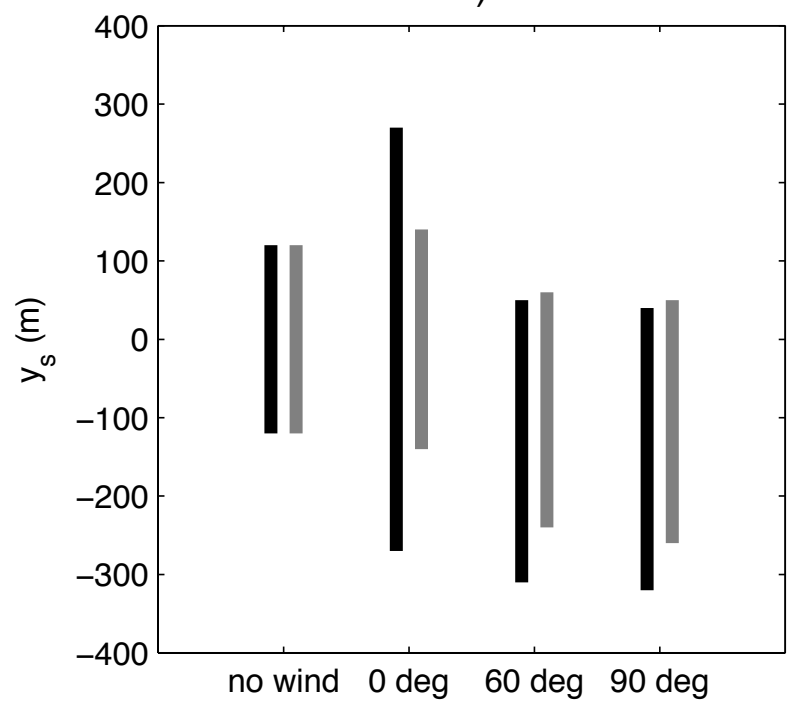

b)

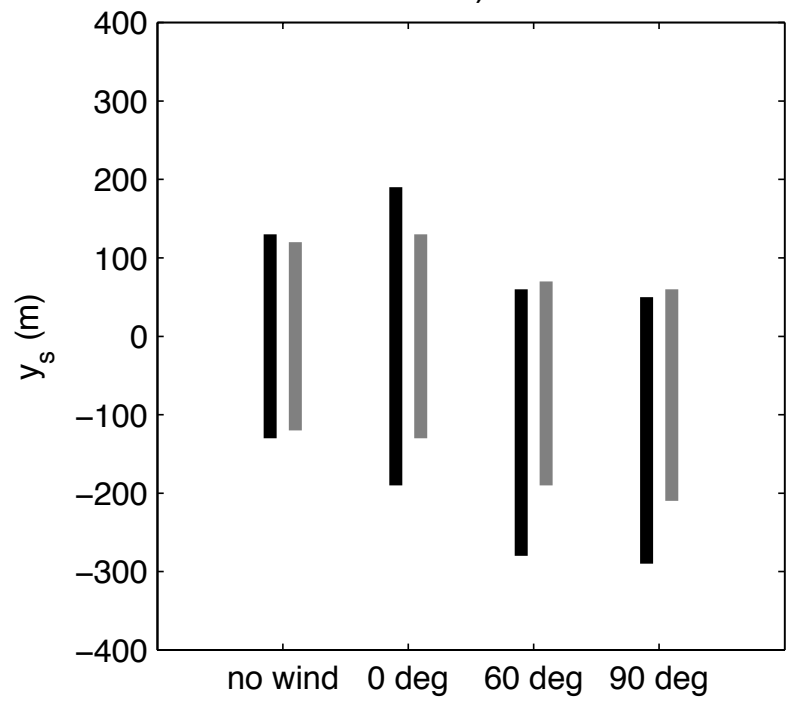

Figure 8. Depiction of the contributing parts of a ILS towards the receiver in case of various wind scenarios, a) receiver height of $1.5 \mathrm{~m}, \mathrm{~b}$ ) receiver height of $4 \mathrm{~m}$. Black: rigid ground surface, Grey: soft ground surface. 